\title{
Dynamic analysis of a stochastically perturbed dysentery diarrhoea epidemic model with controls
}

\author{
Xingwang $\mathrm{Yu}^{1}$ and Yuanlin $\mathrm{Ma}^{1}$ \\ ${ }^{1}$ Zhengzhou University of Aeronautics
}

September 27, 2020

\begin{abstract}
To understand the transmission dynamics of diarrhea in random environment, in this paper we propose a stochastically perturbed dysentery diarrhoea epidemic model with controls. Using the theory of stopping time, we first show the existence of global positive solution of the model. Then, we study the stochastic dynamics of the model and present a stochastic threshold $\$ \backslash$ mathcal $\{\mathrm{R}\} \_0^{\wedge} \mathrm{S} \$$ which determines the extinction and persistence of the disease. Based on Khasminskii's theory, we further prove that the model has a unique ergodic stationary distribution under the condition of $\$ \backslash$ mathcal $\{\mathrm{R}\}\}_{-} 0^{\wedge} \mathrm{S}>1 \$$. Numerical simulations are carried out to verify the analytical results, showing that the white noise, and the constant treatment and sanitation may have certain inhibitory effects on disease transmission. Lastly, the model is further extended to include colored noise and seasonal fluctuation to study the long-term transmission dynamics of disease. It is found that the method proposed in this paper is universal.
\end{abstract}

\section{Hosted file}

MMAS.pdf available at https://authorea.com/users/362229/articles/483451-dynamic-analysis-ofa-stochastically-perturbed-dysentery-diarrhoea-epidemic-model-with-controls 